\title{
Paclitaxel attenuates renal interstitial fibroblast activation and interstitial fibrosis by inhibiting STAT3 signaling
}

This article was published in the following Dove Press journal:

Drug Design, Development and Therapy

I5 April 2015

Number of times this article has been viewed

\author{
Lei Zhang ${ }^{1,2, *}$ \\ Xuan $\mathrm{Xu}^{\mathrm{l}, *}$ \\ Ruhao Yangl,* \\ Jingwen Chen' \\ Shixuan Wang ${ }^{5}$ \\ Junqin Yang ${ }^{3}$ \\ Xudong Xiang' \\ Zhibiao $\mathrm{He}^{\prime}$ \\ Yu Zhao ${ }^{4}$ \\ Zheng Dong 2,5 \\ Dongshan Zhang' \\ 'Department of Emergency \\ Medicine, ${ }^{2}$ Department of Nephrology, \\ ${ }^{3}$ Department of Minimally Invasive \\ Surgery, Second Xiangya Hospital, \\ Central South University, Changsha, \\ Hunan, People's Republic of China; \\ ${ }^{4}$ Department of Nephrology, Harbin \\ First Hospital, Harbin, Heilongjiang, \\ People's Republic of China; \\ ${ }^{5}$ Department of Cellular Biology and \\ Anatomy, Medical College of Georgia \\ at Georgia Regents University and \\ Charlie Norwood VA Medical Center, \\ Augusta, GA, USA \\ *Co-first authors in this study
}

Correspondence: Dongshan Zhang Department of Emergency Medicine, Second Xiangya Hospital, Central South University, 139 Renmin Road, Changsha, Hunan 4I00I I, People's Republic of China

Tel +86 I38 75899625

Email zhkidney@qq.com
Abstract: Recent studies have demonstrated that paclitaxel might inhibit renal fibrosis. However, the underlying molecular mechanism remains unclear. In this study, we hypothesized that low-dose paclitaxel may block the STAT3 (signal transducer and activator of transcription 3) signaling to attenuate fibrosis in a mouse model with unilateral ureteral obstruction. Both NRK49F cells and mice with unilateral ureteral obstruction were treated with paclitaxel. The results showed that paclitaxel treatment resulted in a dose- and time-dependent decrease in tyrosinephosphorylated STAT3, and inhibited the expression of fibronectin, alpha-smooth muscle actin ( $\alpha$-SMA), and collagen I in cultured NRK-49F cells. S3I-201, an STAT3 inhibitor, also suppressed the expression of fibronectin, $\alpha$-SMA, and collagen I in cultured NRK-49F cells. Mechanistically, paclitaxel treatment blocked the STAT3 activity by disrupting the association of STAT3 with tubulin and inhibiting STAT3 nucleus translocation. Furthermore, paclitaxel also ameliorated renal fibrosis by down-regulating the expression of fibronectin, $\alpha$-SMA, and collagen I, and suppressed the infiltration of macrophages and production of TNF- $\alpha$, IL-1 $\beta$, TGF- $\beta$, and ICAM-1 (intercellular adhesion molecule 1) by inhibition of STAT3 activity in obstructive nephropathy. These results suggest that paclitaxel may block the STAT3 activity by disrupting the association of STAT3 with tubulin and inhibiting STAT3 nucleus translocation, consequently leading to the suppression of renal interstitial fibroblast activation and the development of renal fibrosis, and inhibition of proinflammatory cytokine production.

Keywords: UUO, tubulointerstitial fibrosis, tubulin, paclitaxel, STAT3

\section{Introduction}

Paclitaxel, one of the most important anticancer drugs, has been used in the treatment of different types of cancers. Recently, it has been found that paclitaxel could be promising in treating noncancer diseases. ${ }^{1}$ For example, Zhang et $\mathrm{al}^{2}$ reported that paclitaxel significantly suppressed tubulointerstitial fibrosis by inhibiting TGF- $\beta$ (transforming growth factor-beta)/Smad signaling in a rat model of unilateral ureteral obstruction (UUO). Karbalay-Doust et $\mathrm{al}^{3}$ also found that paclitaxel was more effective than taurine in suppressing renal fibrosis in the UUO model. Furthermore, paclitaxel showed an antifibrosis role by blocking TGF- $\beta / \mathrm{Smad} / \mathrm{miR}-192$ signaling. ${ }^{4}$ However, the underlying molecular mechanism is not fully understood.

Renal interstitial fibrosis is a progressive process. The key step is the transformation of the renal fibroblasts to alpha-smooth muscle actin ( $\alpha$-SMA)-positive myofibroblasts in the evolution of chronic kidney disease. ${ }^{5,6}$ Signal transducer and activator of transcription 3 (STAT3) is an important member of the STAT family (STAT14, STAT5a/5b, and STAT6) and mediates cell survival and proliferation. ${ }^{7-9}$ Multiple growth factors and cytokines may activate STAT3 tyrosine phosphorylation that 
promotes STAT3 to form dimers and translocate to the cell nucleus to regulate the transcription of target genes. ${ }^{7-9}$ It has been reported that STAT3 activation mediates the activation of renal interstitial fibroblasts and the progression of renal fibrosis in UUO models. ${ }^{10,11}$ Interestingly, Walker et al ${ }^{12}$ reported that paclitaxel might inhibit STAT3 signaling in several tumor cell lines. In view of these findings, this study was initiated to assess whether paclitaxel can, by blocking STAT3 signaling, attenuate the activation of renal interstitial fibroblasts and the progression of renal fibrosis.

\section{Materials and methods}

\section{Reagents and antibodies}

S3I-201 was purchased from Calbiochem (La Jolla, CA, USA). Antibodies were obtained from different sources: anti-GAPDH, anti- $\alpha-S M A$, anticollagen I, and antifibronectin from Santa Cruz Biotechnology (Santa Cruz, CA, USA), antimacrophage and Gr-1 from Abcam (Cambridge, MA, USA), anti- $\alpha$-tubulin from Sigma-Aldrich (St Louis, MO, USA), anti-STAT3 and anti-p-STAT3 from Cell Signaling Technology (Danvers, MA, USA). The kit for protein isolation of cytoplasm and nucleus was purchased from NobleRyde (Beijing, People's Republic of China). All secondary antibodies were from Thermo Fisher Scientific (Waltham, MA, USA).

\section{Cell culture and treatments}

NRK-49F cells were cultured in Dulbecco's modified Eagle's medium (Sigma-Aldrich) supplemented with 10\% fetal bovine serum, $0.5 \%$ penicillin, and streptomycin in an atmosphere of $5 \% \mathrm{CO}_{2}$ and $95 \%$ air at $37^{\circ} \mathrm{C}$.

\section{Care and use of laboratory animals}

Animal experiments were performed in accordance with the regulations set by the Institutional Committee for the Care and Use of Laboratory Animals of Second Xiangya Hospital, People's Republic of China, and approved by local authorities. C57BL/6 mice were housed on a 12-hour light/dark cycle and were allowed free access to food and water.

\section{Animal model}

The UUO model was established in male C57 black mice that weighed 20-25 g (Shanghai animal center, Shanghai, People's Republic of China), as previously described. ${ }^{2}$ Four groups of mice comprising eight animals each (total 32) were divided as follows: 1) Sham group, 2) Sham with paclitaxel (Taxol; Sigma-Aldrich) group, 3) UUO group, and 4) UUO with paclitaxel group. Paclitaxel was injected intraperitoneally at a dose of $0.3 \mathrm{mg} / \mathrm{kg}$ twice a week. The control group was administered with saline. Mice were sacrificed on day 7 after UUO or sham operation. The kidneys were harvested for various biochemical and morphological studies.

\section{Immunoprecipitation}

The immunoprecipitation was performed as previously described. ${ }^{12,13}$ Briefly, approximately $500 \mu \mathrm{g}$ of cellular protein was immunoprecipitated with $2 \mu \mathrm{g}$ of antibodies to STAT3 or $\alpha$-tubulin for 1 hour at $4^{\circ} \mathrm{C}$, followed by the addition of $15 \mu \mathrm{L}$ of protein A/G-agarose beads (Santa Cruz Biotechnology). The precipitates were washed four times with lysis buffer and subjected to immunoblotting for p-STAT3 and STAT3.

\section{Histology and immunohistochemistry}

For the histological analysis, kidney tissues fixed with $4 \%$ buffered paraformaldehyde were embedded in paraffin and $3 \mu \mathrm{m}$ thick sections were prepared. The sections were then stained with hematoxylin-eosin and Masson's trichrome methods. ${ }^{2}$ The interstitial fibrosis and interstitial gross area were separately analyzed by an image analysis software of Image-Pro Plus 6 (Media Cybernetics, Inc., Bethesda, MD, USA); then we calculated the percent positive area of renal interstitial fibrosis versus renal interstitial gross area. Immunohistochemical analyses were performed using antiphosphoSTAT3, anti- $\alpha$-SMA, anticollagen I, antifibronectin, and antimacrophage according to the previous protocol. ${ }^{2,14}$ The total number of positive cells for p-STAT3 (as identified by nuclear staining) and macrophage in tubulointerstitium was quantified by counting the number of stained cells per field, for p-STAT3. We collected 25-30 images of a kidney from each animal at $20 \times$ magnification. Areas of positive staining for $\alpha$-SMA collagen type I and fibronectin in the entire cortical tubulointerstitium (a cross section of the kidney) were examined using quantitative software of Image-Pro Plus 6 (Media Cybernetics, Inc). Briefly, the detected area of tubulointerstitium was labeled, the positive-staining parts were discriminated, and the percent positive area in the detected tubulointerstitium was then measured. ${ }^{2,14}$

\section{Immunoblot analysis}

Immunoblot was carried out as previously described. ${ }^{14}$ Briefly, cells or kidney tissues were treated with a lysis buffer (SigmaAldrich) containing phosphatase inhibitors (Calbiochem). Equal amounts of proteins were loaded in each well for electrophoresis using SDS-PAGE gel, followed by transferring to polyvinylidene fluoride membranes. The blots were incubated 
with primary antibodies overnight at $4^{\circ} \mathrm{C}$ and probed by the horseradish peroxidase-conjugated secondary antibodies. Bands of target and internal control protein were separately outlined, and then gray level was analyzed by an image J software (NIH, Bethesda, MD, USA); then we calculated gray ratio of target protein versus internal control protein.

\section{Measurement of TNF- $\alpha$, IL-I $\beta$, and TGF- $\beta$}

The levels of TNF- $\alpha$ (tumor necrosis factor- $\alpha$ ), IL- $1 \beta$ (interleukin-1 $\beta$ ), and TGF- $\beta$ were determined using enzymelinked immunosorbent assay (ELISA) kits (R\&D Systems Inc, Minneapolis, MN, USA) as described previously. ${ }^{11,13}$ Renal cytokine levels were normalized by the protein concentration.

\section{Real-time polymerase chain reaction}

Real-time quantitative reverse transcriptase PCR amplifications were performed in $20 \mu \mathrm{L}$ reactions as described previously. ${ }^{4}$ The primer sets used for various genes were as follows: ICAM-1 (intercellular adhesion molecule-1): forward 5'-CTTCCAGCTACCATCCCAAA-3', reverse 5'-CTTCAGAGGCAGGAAACAGG-3'; GADPH: forward 5'-TGCTGAGTATGTCGTGGAGTCTA-3', reverse 5'-AGTGGGAGTTGCTGTTG AAATC-3'.

\section{Statistical analysis}

Data were calculated as mean \pm SEM (standard error of the mean). One-way ANOVA, followed by the Tukey's post hoc test, was used to compare multiple treatment groups. Two-way ANOVA was used to assess the statistical significance of the differences between multiple treatment groups at different time points. Statistical significance was set at $P<0.05$ or $<0.01$.

\section{Results}

\section{Paclitaxel suppresses the expression of fibronectin, $\alpha$-SMA, and collagen I by inhibiting STAT3 activation in cultured renal interstitial fibroblasts}

Previous results have demonstrated that STAT3 mediated the activation of renal interstitial fibroblasts, characterized by the expression of fibronectin, $\alpha$-SMA, and collagen I. ${ }^{11,15}$ To further investigate whether low-dose paclitaxel suppresses the activation of renal interstitial fibroblasts by inhibiting STAT3 activation, we examined the effect of paclitaxel on STAT3 activation and expression of fibronectin, $\alpha$-SMA, and collagen I in NRK-49F cells. As shown in Figure 1A-D, treatment with paclitaxel resulted in a dose- and timedependent decrease in tyrosine-phosphorylated STAT3 in NRK-49F cells. Furthermore, the expression level of fibronectin, $\alpha$-SMA, and collagen I was higher in NRK49F cells (Figure 1E and F), indicating that the cultured renal interstitial fibroblasts had the phenotypic features of myofibroblasts. Treatment with paclitaxel also significantly decreased the expression of fibronectin, $\alpha-S M A$, and collagen $I$ in a time-dependent manner. This result suggests that paclitaxel suppresses the activation of renal interstitial fibroblasts by inhibiting STAT3 activation.

\section{Paclitaxel blocks tyrosine-phosphorylated STAT3 (Tyr705) by inhibiting the association of STAT3 with tubulin and STAT3 nucleus translocation}

On the basis of the findings that STAT3 directly interacted with tubulin and that paclitaxel was a microtubule stabilization agent, we presume that paclitaxel inhibits STAT3 activation by interfering with its association with tubulin. NRK-49F cells were treated with vehicle, paclitaxel, or S3I-201, which is a novel selective inhibitor of STAT3. ${ }^{16}$ First, we confirmed that S3I-201 suppressed the expression of fibronectin, $\alpha$-SMA, and collagen I by blocking STAT3 activation (Figure 2A and B). However, S3I-201 also increased the expression of p-STAT1 but did not affect that of p-STAT5 at the concentration of $50 \mu \mathrm{M}$ (data not shown). Briefly, the cell lysate was immunoprecipitated with an anti-STAT3 antibody, followed by the immunoblot detection of STAT3 tyrosine phosphorylation (Tyr705). Both paclitaxel and S3I-201 inhibited STAT3 tyrosine phosphorylation (Tyr705) (Figure 2C and D). We further examined the effect of paclitaxel on STAT3/tubulin association by immunoprecipitation assay. The cell lysate was immunoprecipitated with an antitubulin antibody, followed by the immunoblot detection of STAT3. IgG was used as the input control. The association of STAT3 with tubulin was suppressed by paclitaxel treatment (Figure 2E and F). However, S3I-201 treatment did not affect the association between STAT3 and tubulin. Thus, paclitaxel disrupted the association of STAT3 with tubulin, independently of the effect on STAT3 phosphorylation. Because paclitaxel might inhibit the STAT3 binding to tubulin, we presume that paclitaxel might suppress STAT3 translocation. Proteins of cytoplasm and nucleus from kidneys were separately extracted using a commercial kit, followed by immunoblot. The results demonstrated that paclitaxel blocked STAT3 
A

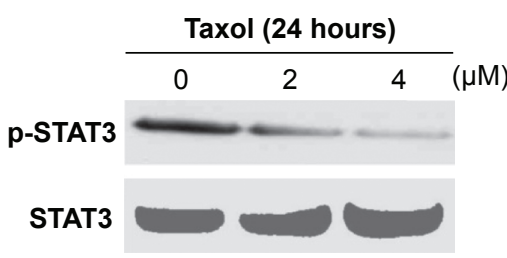

C

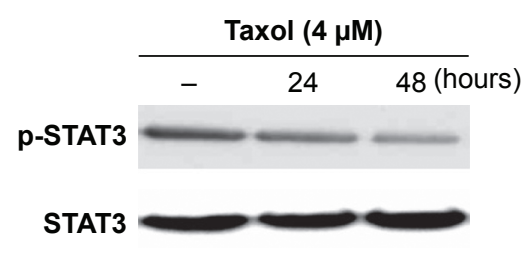

E

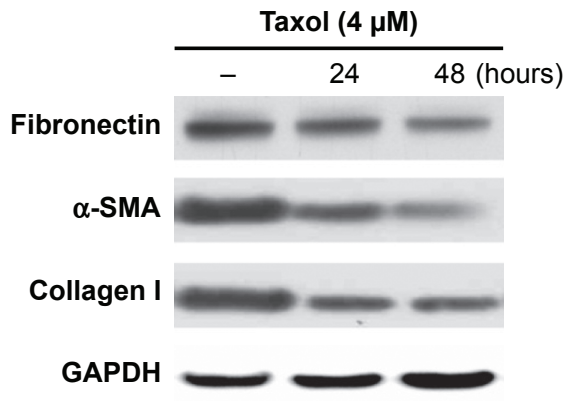

B

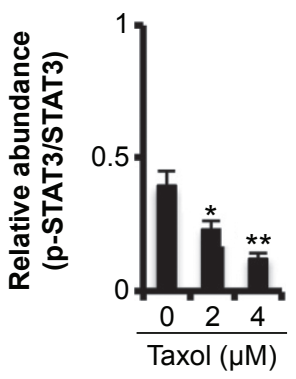

D

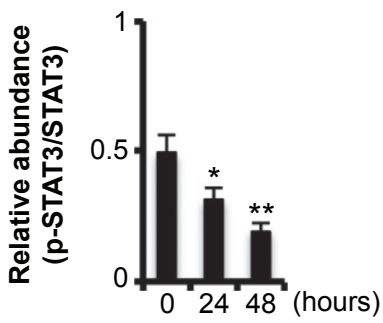

F

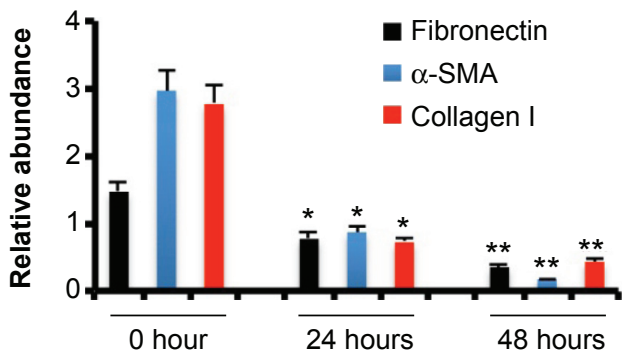

Figure I Effect of paclitaxel on STAT3 activation and the expression of fibronectin, alpha-smooth muscle actin ( $\alpha$-SMA), and collagen I.

Notes: Cultured NRK-49F cells were treated with paclitaxel of the indicated concentrations for 24 hours, or exposed to $4 \mu M$ paclitaxel for the indicated time period. (A) Immunoblot analysis of p-STAT3 and STAT3 after 24 hours of treatment with $2 \mu M$ and $4 \mu$ M paclitaxel. (B) P-STAT3/STAT3 ratio was found to be activated STAT3 using gray analysis. (C) Immunoblot analysis of p-STAT3 and STAT3 after 24 and 48 hours of treatment with $4 \mu M$ paclitaxel. (D) p-STAT3/STAT3 ratio was indicated as activated STAT3 by gray analysis. (E) Immunoblot analysis of fibronectin, $\alpha$-SMA, collagen I, and GAPDH after 24 and 48 hours of treatment with $4 \mu M$ paclitaxel. (F) Expression levels of fibronectin, $\alpha$-SMA, and collagen I were quantified by densitometry, and normalized with GAPDH. Representative immunoblots from each group ( $n=8$ ). Data are presented as mean \pm SEM. Significant $P$-values reflecting differences are indicated over the bars $(* P<0.05 ; * * P<0.01)$.

Abbreviations: STAT3, signal transducer and activator of transcription 3; Taxol, paclitaxel; GAPDH, glyceraldehyde 3-phosphate dehydrogenase; SEM, standard error of the mean; $\alpha$-SMA, alpha-smooth muscle actin.

translocation from the cytoplasm to the nucleus (Figure $2 \mathrm{G}$ and $\mathrm{H})$, which further supported that paclitaxel blocked STAT3 activation by inhibiting the association of STAT3 with tubulin.

\section{Paclitaxel attenuates tubulointerstitial fibrosis in mice obstructive nephropathy}

Previous results demonstrated that paclitaxel attenuated fibrosis in a rat UUO model. In this experiment, we confirmed that paclitaxel also ameliorated tubulointerstitial fibrosis and tubular injury in a mouse UUO model. Briefly, hematoxylineosin staining showed that UUO animals exhibited significant tubular atrophy on day 7 , which was notably reduced with paclitaxel treatment (Figure 3A). Masson's trichrome staining demonstrated that UUO animals exhibited marked tubulointerstitial fibrosis on day 7 , which was significantly reduced by paclitaxel treatment (Figure 3B and C).

\section{Blockade of STAT3 activation is a key mechanism by which paclitaxel prevents renal fibrosis in mouse obstructive nephropathy}

We have demonstrated that paclitaxel suppressed STAT3 activation in vitro. To further investigate whether paclitaxel suppresses STAT3 activation and expression of collagen I, fibronectin, and $\alpha$-SMA in mouse obstructive nephropathy, we examined the effect of paclitaxel. By immunohistochemistry, we found that the expression of p-STAT3, collagen I, fibronectin, and $\alpha$-SMA were markedly increased in UUO than in the Sham group on day 7 , which was significantly 
A

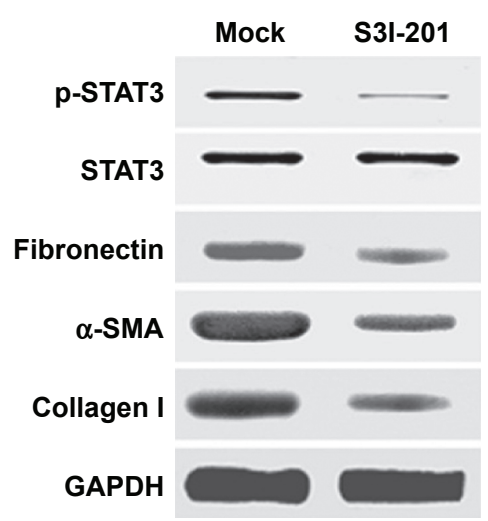

C

E

G

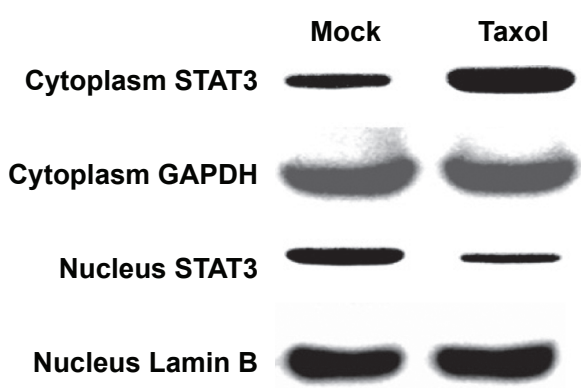

B

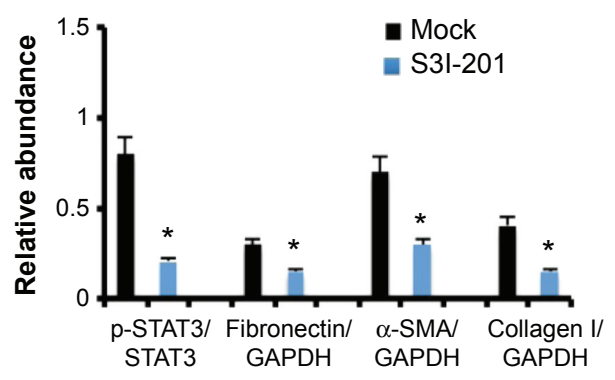

D

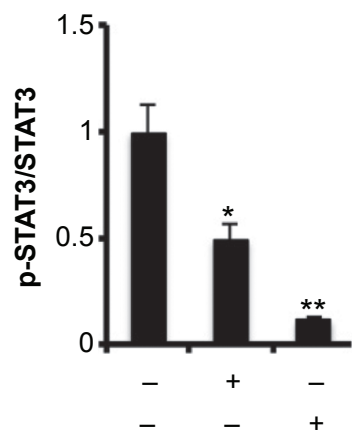

$\mathbf{F}$

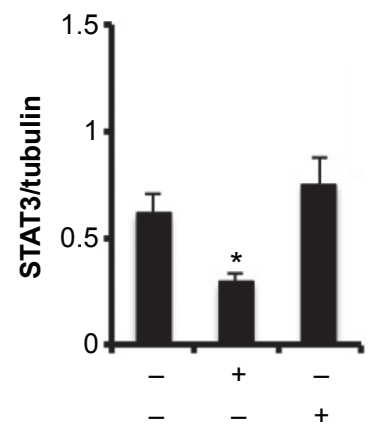

H

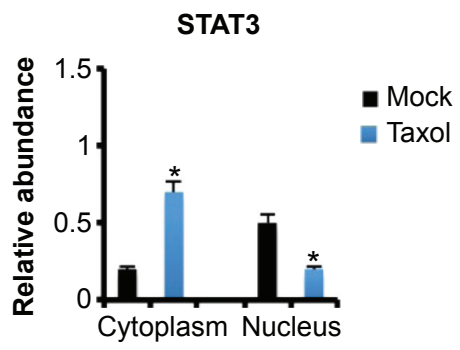

Figure 2 Paclitaxel disrupts interaction of STAT3 with tubulin and inhibits STAT3 nucleus translocation.

Notes: Cultured NRK-49F cells were treated with $4 \mu \mathrm{M}$ paclitaxel or $50 \mathrm{mM} \mathrm{S3I-20I}$ for 6 to 24 hours, followed by immunoblot for STAT3, P-STAT3, and ECM genes, and immunoprecipitation with antibodies to STAT3 or tubulin. (A) Immunoblot analysis of STAT3, p-STAT3, fibronectin, $\alpha$-SMA, collagen I, and GAPDH after 24 hours of treatment with $50 \mathrm{mM} \mathrm{S3I-20I}$. (B) Expression levels of fibronectin, $\alpha$-SMA, and collagen I were quantified by densitometry, and normalized with GAPDH. The density of the bands was quantitated, and P-STAT3 was normalized to STAT3 as a loading control. (C) Immunoprecipitates with antibodies to STAT3 were then subjected to immunoblot analysis of STAT3 and p-STAT3, with IgG as input control. (D) The density of the bands was quantitated, and p-STAT3 was normalized to STAT3 as a loading control. (E) Immunoprecipitates with antibodies to tubulin were then subjected to immunoblot analysis of STAT3, with IgG as input control. (F) The density of the bands was quantitated, and STAT3 was normalized to tubulin as a loading control. (G) Immunoblot analysis of STAT3 in cytoplasm and nucleus at 6 hours of treatment with paclitaxel. (H) The density of the bands was quantitated, and STAT3 was normalized to GAPDH or Lamin B as a loading control. Representative immunoblots from each group ( $=8$ ). Data are presented as mean \pm SEM. Significant $P$-values reflecting differences are indicated over the bars $(* P<0.05 ; * * P<0.0$ I).

Abbreviations: STAT3, signal transducer and activator of transcription 3; $\alpha$-SMA, alpha-smooth muscle actin; Taxol, paclitaxel; GAPDH, glyceraldehyde 3-phosphate dehydrogenase; ECM, extracellular matrix; SEM, standard error of the mean. 


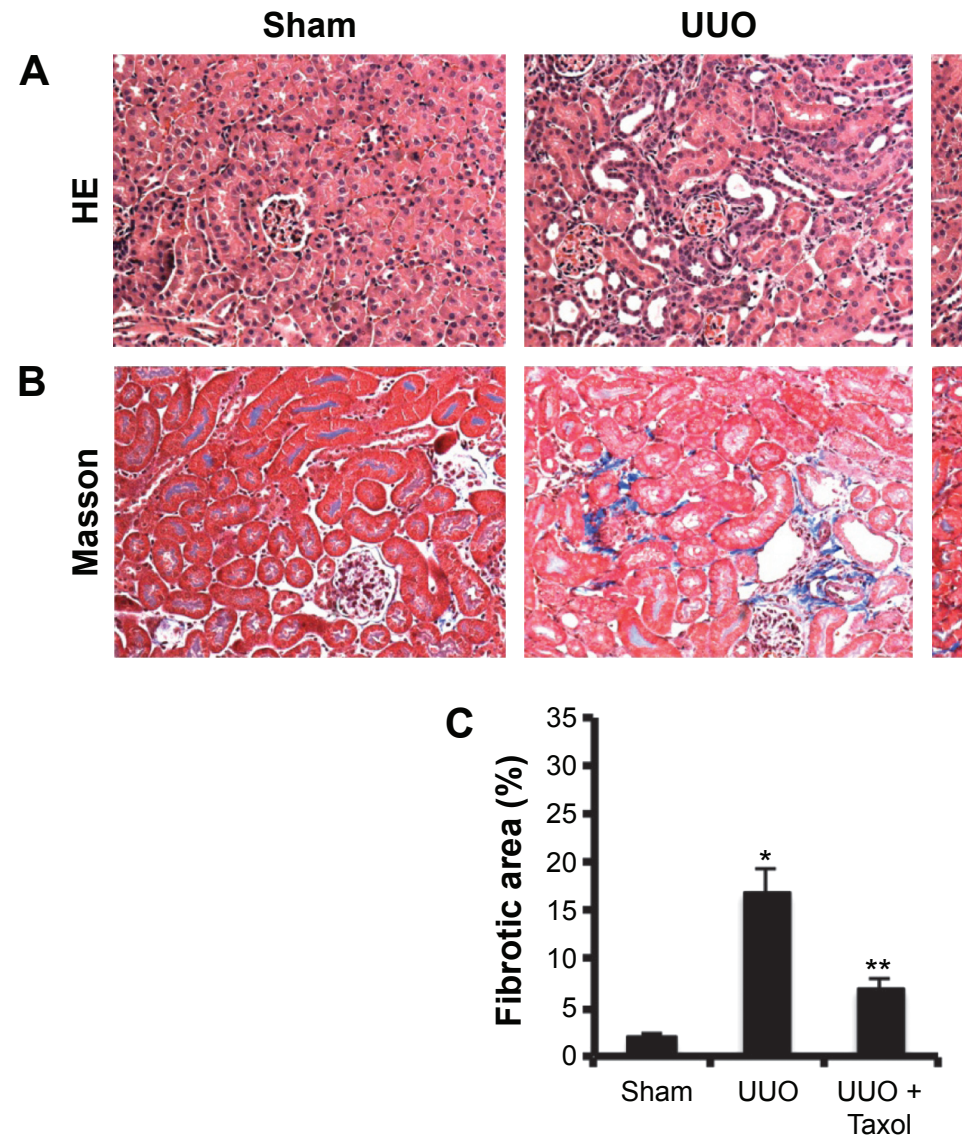

Figure 3 Paclitaxel attenuates tubulointerstitial fibrosis in a mouse UUO model.

Notes: The left ureter of male C57 mice was ligated for 7 days with or without $0.3 \mathrm{mg} / \mathrm{kg}$ paclitaxel twice a week. As a control, the Sham group was injected with saline. Kidney tissues were collected on day 7 after treatment. (A) HE-stained paraffin-embedded mouse kidney sections. (B) Representative Masson's trichrome-stained sections. (C) Semiquantitative scores of tubulointerstitial fibrosis in the kidney cortex. Original magnifications, $\times 200$. Representative immunoblots from each group ( $=8$ ). Data are presented as mean \pm SEM. Significant $P$-values reflecting differences are indicated over the bars $(* P<0.05 ; * * P<0.01)$.

Abbreviations: UUO, unilateral ureteral obstruction; Taxol, paclitaxel; HE, hematoxylin-eosin; SEM, standard error of the mean.

reduced by paclitaxel treatment (Figure 4A-E). Western blot analysis indicated similar results (Figure $4 \mathrm{~F}$ and $\mathrm{G}$ ). These findings suggest that paclitaxel suppresses the activation of renal fibroblasts to attenuate renal fibrosis by inhibiting STAT3 activation in a mouse UUO model, being consistent with in vitro findings.

\section{Effect of paclitaxel on macrophages and neutrophils infiltration and expression of TNF- $\alpha$, IL-I $\beta$, TGF- $\beta$, and ICAM-I in mouse obstructive nephropathy}

We examined the effect of paclitaxel on macrophage and neutrophil infiltration and the expression of TNF- $\alpha$, IL- $1 \beta$, TGF- $\beta$, and ICAM- 1 . Analysis of kidney sections by immunochemistry showed a prominent interstitial infiltration of macrophages and neutrophils after obstructive injury, which was significantly reduced by paclitaxel (Figure 5A-C). The expression levels of TNF- $\alpha$, IL- $1 \beta$, TGF- $\beta$, and ICAM- 1 were increased after obstructive injury, which were also markedly reduced by paclitaxel (Figure 6A-D). These data suggest that paclitaxel suppresses macrophage and neutrophil infiltration and proinflammatory cytokines by inhibiting STAT3 activity in UUO-induced renal fibrosis.

\section{Discussion and conclusion}

Previous results demonstrated that paclitaxel might ameliorate renal fibrosis in a rat UUO model. ${ }^{2}$ However, the mechanism remains unclear. In this study, the results show that paclitaxel treatment inhibits the expression of $\alpha$-SMA and extracellular matrix proteins in renal interstitial fibroblasts, ameliorates renal fibrosis, and suppresses the infiltration of macrophages and proinflammatory cytokines in obstructive nephropathy.

Mechanistically, we observed that paclitaxel treatment blocked the STAT3 activity in cultured renal interstitial fibroblasts and the fibrotic mouse kidney. TGF- $\beta 1$, plateletderived growth factor (PDGF), and IL-6 can induce STAT3 

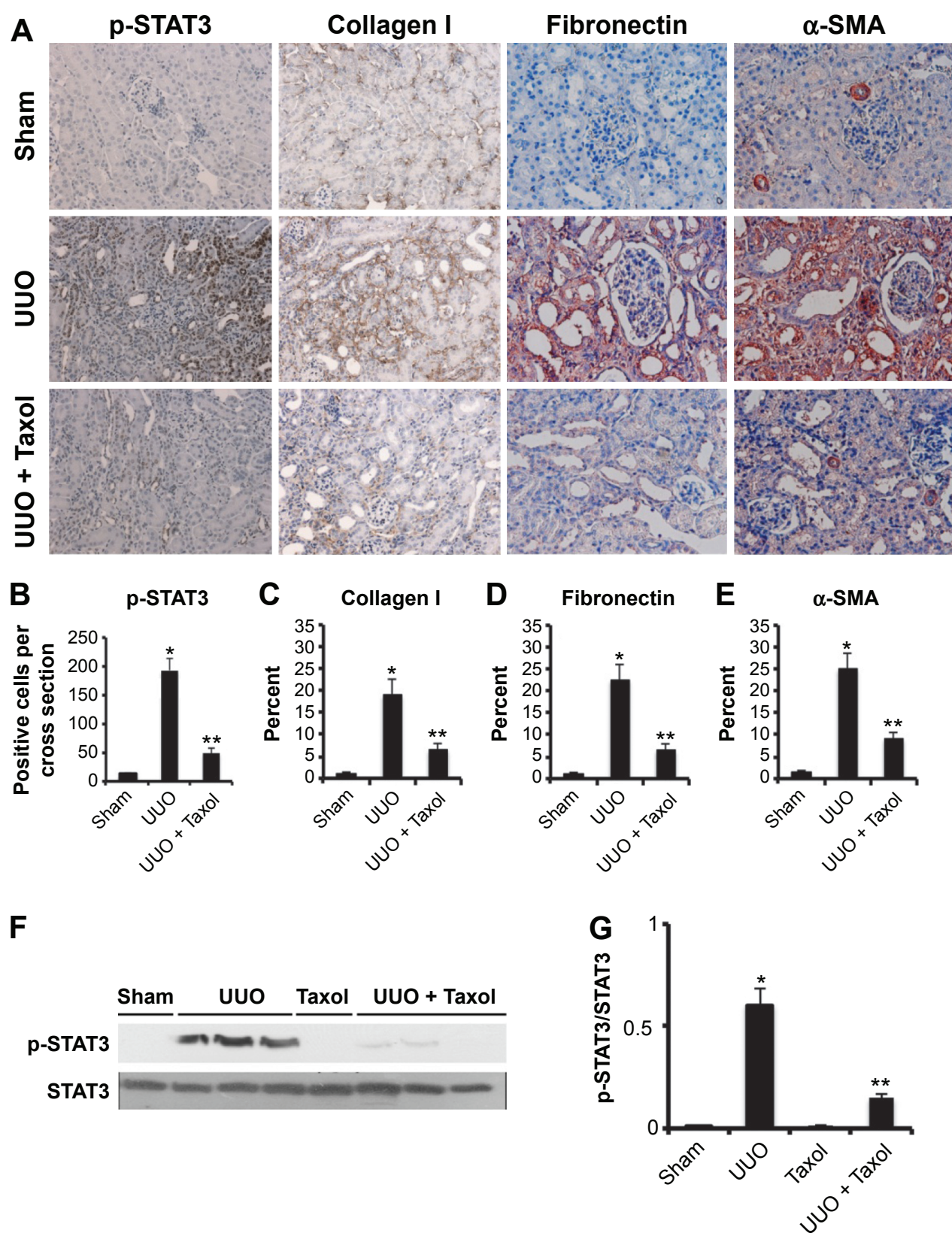

Figure 4 Paclitaxel inhibits STAT3 activation and collagen I, fibronectin, and $\alpha$-SMA in a mouse UUO model.

Notes: The left ureters of male C57 mice were ligated for 7 days with or without $0.3 \mathrm{mg} / \mathrm{kg}$ paclitaxel twice a week. As a control, the Sham group was injected with saline. Kidney tissues were collected on day 7 after treatment. (A) Representative $\mathrm{p}$-STAT3, collagen I, fibronectin, and $\alpha$-SMA immunohistochemistry (original magnification, $\times 200$ or $\times 400$ ). (B-E) Densitometric analyses were performed for each group $(n=8)$. (F) Immunoblot analysis of $p-S T A T 3$ and STAT3 from mice kidneys of different groups on day 7 . (G) p-STAT3/STAT3 ratio was indicated as activated STAT3 using gray analysis. Representative immunoblots from each group ( $\mathrm{n}=8$ ). Data are presented as mean \pm SEM. Significant $P$-values reflecting differences are indicated over the bars $(* P<0.05 ; * * P<0.01)$.

Abbreviations: STAT3, signal transducer and activator of transcription 3; $\alpha$-SMA, alpha-smooth muscle actin; UUO, unilateral ureteral obstruction; Taxol, paclitaxel; SEM, standard error of the mean.

activation, ${ }^{17-20}$ which was detected in several human diseases associated with the development of fibrosis, including liver fibrosis, scar formation after spinal cord injury, glomerulonephritis, and diabetic nephropathy. ${ }^{9,21-25}$ Furthermore, Pang et $\mathrm{a}^{11}$ reported that STAT3 played an important role in mediating the activation of renal interstitial fibroblasts and the development of renal fibrosis following UUO injury.
In this study, we demonstrated that paclitaxel ameliorated fibrosis by inhibiting STAT3 activity in cultured renal interstitial fibroblasts and mouse UUO models. We wanted to know how the microtubule-targeting agents inhibit STAT3 activation. Mechanistically, the interaction between STAT3 and microtubules was prevented by paclitaxel. Paclitaxel may also have an effect on nonphosphorylated 


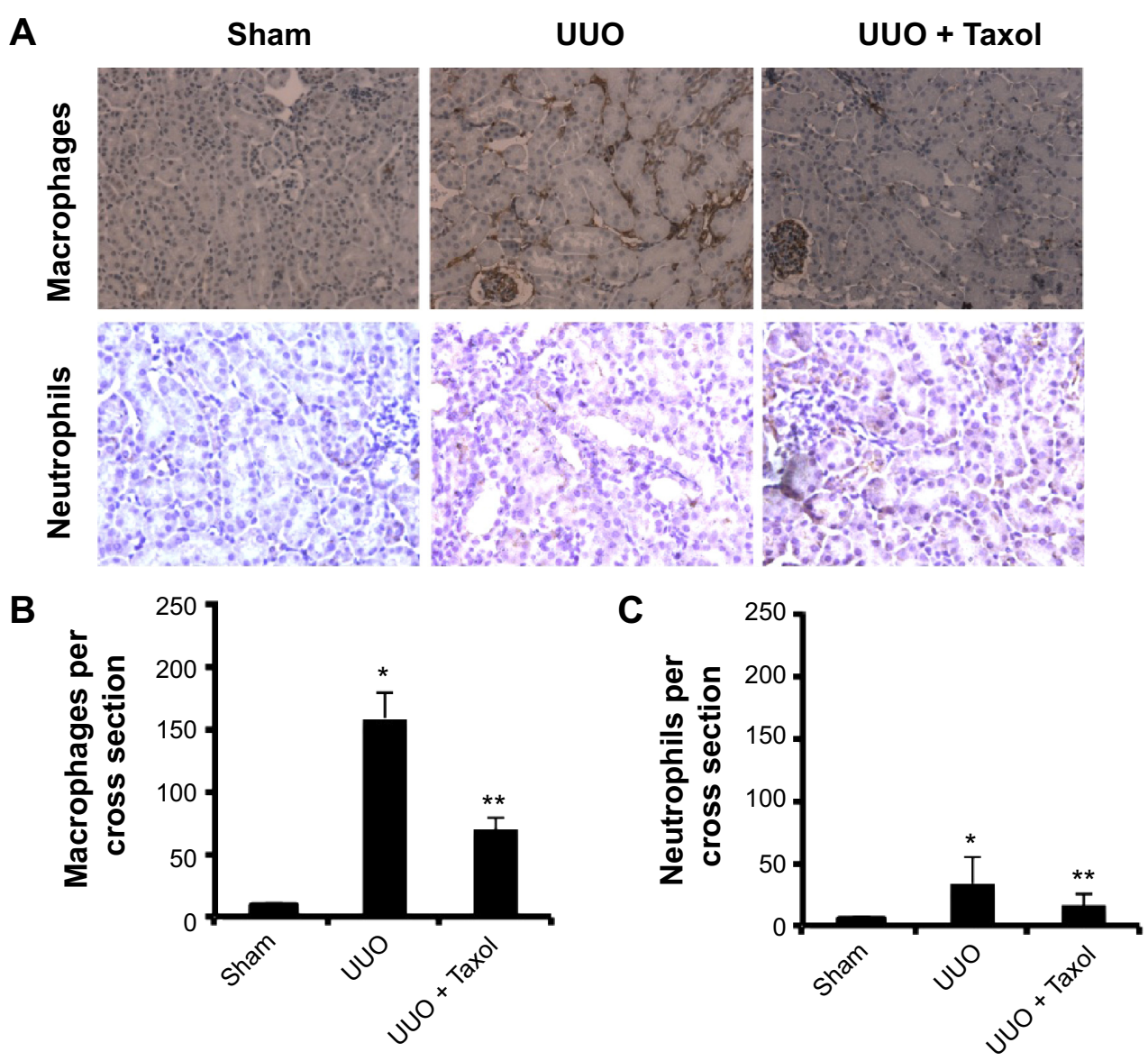

Figure 5 Paclitaxel inhibits infiltration of macrophages and neutrophils in a mouse UUO model.

Notes: The left ureters of male C57 mice were ligated for 7 days with or without $0.3 \mathrm{mg} / \mathrm{kg}$ paclitaxel twice a week. As a control, the Sham group was injected with saline. Kidney tissues were collected on day 7 after treatment. (A) Representative macrophage and neutrophil immunohistochemistry from kidney cortical tissues of different groups on day 7 (original magnification, $\times 400$ ). (B and $\mathbf{C}$ ) Quantitation of macrophage- and neutrophil-positive cells per total cross-sectional area was performed from each group $(n=8)$. Data are presented as mean \pm SEM. Significant $P$-values reflecting differences are indicated over the bars $(* P<0.05 ; * * P<0.01)$.

Abbreviations: UUO, unilateral ureteral obstruction; Taxol, paclitaxel; SEM, standard error of the mean.

forms of STAT3, thereby preventing their translocation to the nucleus. Walker et al ${ }^{12}$ demonstrated that phosphorylated STAT3 was significantly inhibited by paclitaxel at a concentration of $7 \mu \mathrm{M}$ in MDA-MB-468, MDA-MB-231, and MCF-7 cell lines. Interestingly, paclitaxel at concentrations $2 \mu \mathrm{M}$ or $4 \mu \mathrm{M}$ also blocked STAT3 activation in renal interstitial fibroblasts, which indicated that different cell lines had different levels of sensitivity for paclitaxel treatment. However, paclitaxel at concentration $4 \mu \mathrm{M}$ was a relatively high dose in most cell lines according to value of the $50 \%$ inhibitory concentration $\left(\mathrm{IC}_{50}\right)$. Furthermore, paclitaxel as a chemotherapeutic agent is usually used at a dosage range from 10 to $15 \mathrm{mg} / \mathrm{kg}$ in nude mice. ${ }^{26,27}$ Consequently, paclitaxel at $0.3 \mathrm{mg} / \mathrm{kg}$ in the UUO model is lower than the dosages used in nude mice, but we found that paclitaxel at this dose also markedly inhibited p-STAT3 activation.

Inflammatory responses could accelerate the progression of renal fibrosis, in which multiple inflammatory mediators play a pivotal role. ${ }^{28,29}$ Pang et $\mathrm{al}^{11}$ reported that inhibition of STAT3 activity by S3I-201 could reduce the infiltration of neutrophils and monocytes in the obstructed kidney. In this study, we observed that the administration of paclitaxel also inhibited macrophage infiltration and the proinflammatory cytokines TNF- $\alpha$, IL- $1 \beta$, TGF- $\beta$, and ICAM- 1 . Zhang et $\mathrm{al}^{30}$ demonstrated that STAT3 activity in monocytes/macrophages was a critical mediator of macrophage migration, which supported our results. As we know, TGF- $\beta$ is the major cytokine in a variety of fibrogenic processes, which initiates the transition of renal tubular epithelial cells to myofibroblasts, the cellular source for extracellular matrix synthesis. ${ }^{31,32}$ Previous results have shown that low-dose paclitaxel can significantly suppress the exacerbated TGF- $\beta /$ Smad signaling in the kidney and lessen the interstitial fibrosis in the rat UUO model. ${ }^{2}$ STAT3 and Smad3 are independent signaling pathways for the regulation of fibrosis development. Ogata et $\mathrm{al}^{9}$ reported that STAT3 might directly regulate TGF- $\beta$ 
A

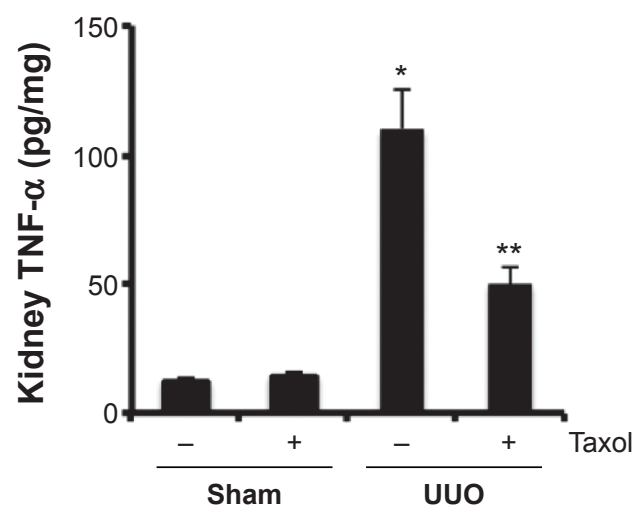

C

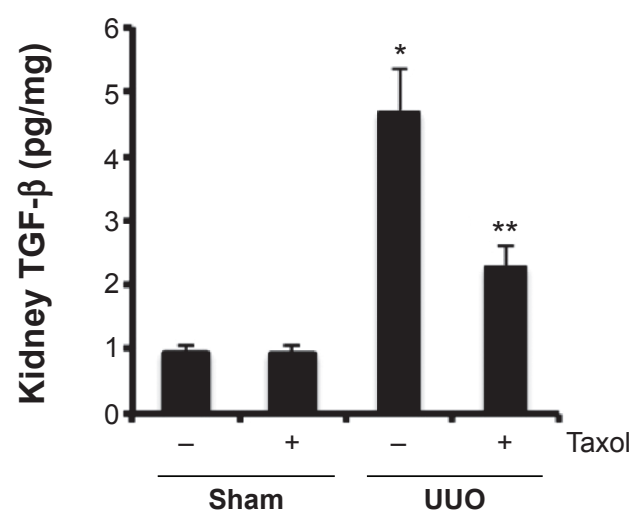

B

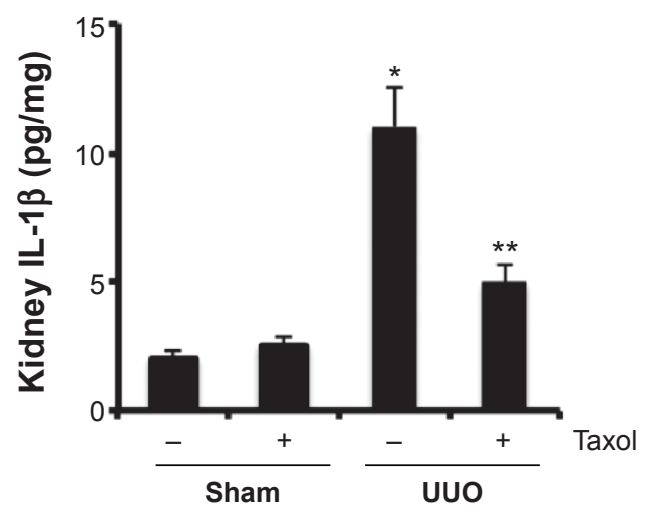

D

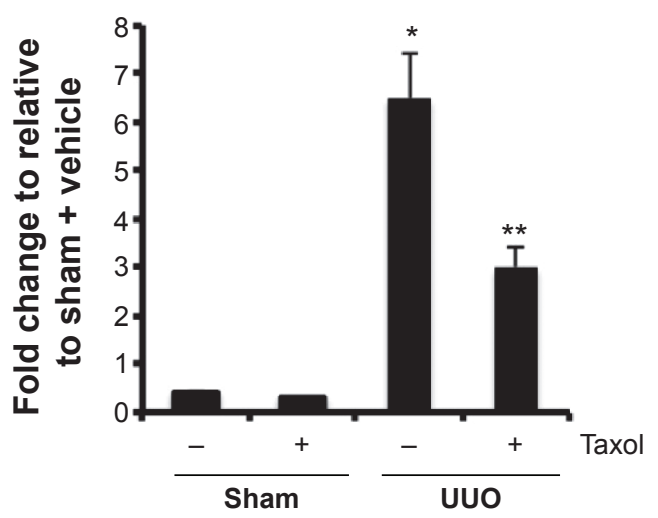

Figure 6 Effect of paclitaxel on the expression of TNF- $\alpha$, IL-I $\beta$, TGF- $\beta$, and ICAM-I.

Notes: The left ureters of male C57 mice were ligated for 7 days with or without $0.3 \mathrm{mg} / \mathrm{kg}$ paclitaxel twice a week. As a control, the Sham group was injected with saline. Kidney tissues and blood samples were collected on day 7 after treatment for determination of TNF- $\alpha$ (A), IL-I $\beta$ (B), and TGF- $\beta$ (C) by ELISA and for real-time PCR determination of ICAM-I (D). ELISA analyses were performed for each group $(n=8)$. Data are presented as mean \pm SEM. Significant $P$-values reflecting differences are indicated over the bars $(* P<0.05 ; * * P<0.01)$.

Abbreviations: TNF- $\alpha$, tumor necrosis factor- $\alpha$; UUO, unilateral ureteral obstruction; Taxol, paclitaxel; IL-I $\beta$, interleukin-I $\beta$; TGF- $\beta$, transforming growth factor-beta; ICAM-I, intercellular adhesion molecule-I; ELISA, enzyme-linked immunosorbent assay; PCR, polymerase chain reaction; SEM, standard error of the mean.

gene expression. In our study, TGF- $\beta$ expression is also reduced by paclitaxel. These results suggest that paclitaxel inhibition on inflammatory responses was another mechanism of anti-renal fibrosis.

In conclusion, our study demonstrates that paclitaxel can suppress the activation of renal fibroblasts, progression of renal fibrosis, and proinflammatory response in UUO kidney injury, suggesting a potential use of the chemotherapy drug in the treatment of renal fibrotic diseases. Mechanistically, paclitaxel treatment blocks the STAT3 activity by disrupting the association of STAT3 with tubulin and STAT3 nucleus translocation.

\section{Acknowledgment}

The study was supported in part by grants from the National Natural Science Foundation of China (81100507/H0509) and the Central South University Master Freedom Investigation program (2014zzts354).

\section{Author contributions}

$\mathrm{DZ}$ and ZD conceived and designed the experiments; LZ, RY, JY, and XX carried out the experiments; JC, YZ, and $\mathrm{ZH}$ analyzed the data; XX, LZ, and RY contributed reagents/ materials/analysis tools; DZ wrote the paper. All authors contributed toward data analysis, drafting and revising the paper and agree to be accountable for all aspects of the work.

\section{Disclosure}

The authors report no conflicts of interest in this work.

\section{References}

1. Zhang D, Yang, R, Wang S, Dong Z. Paclitaxel: new uses for an old drug. Drug Des Devel Ther. 2014;20(8):279-284.

2. Zhang D, Sun L, Xian W, et al. Low-dose paclitaxel ameliorates renal fibrosis in rat UUO model by inhibition of TGF- $\beta /$ Smad activity. Lab Invest. 2010;90(3):436-447.

3. Karbalay-Doust S, Noorafshan A, Pourshahid SM. Taxol and taurine protect the renal tissue of rats after unilateral ureteral obstruction: a stereological survey. Korean J Urol. 2012;53(5):360-367. 
4. Sun L, Zhang D, Liu F, et al. Low-dose paclitaxel ameliorates fibrosis in the remnant kidney model by down-regulating miR-192. J Pathol. 2011;225(3):364-377.

5. Wynn TA. Cellular and molecular mechanisms of fibrosis. JPathol.2008; 214:199-210.

6. Neilson EG. Mechanisms of disease: fibroblasts-a new look at an old problem. Nat Clin Pract Nephrol. 2006;2(2):101-108.

7. Horvath CM. STAT proteins and transcriptional responses to extracellular signals. Trends Biochem Sci. 2000;25(10):496-502.

8. Fuller GM, Zhang Z. Transcriptional control mechanism of fibrinogen gene expression. Ann N Y Acad Sci. 2001;936:469-479.

9. Ogata H, Chinen T, Yoshida T, et al. Loss of SOCS3 in the liver promotes fibrosis by enhancing STAT3-mediated TGF-beta1 production. Oncogene. 2006;25(17):2520-2530.

10. Kuratsune M, Masaki T, Hirai T, et al. Signal transducer and activator of transcription 3 involvement in the development of renal interstitial fibrosis after unilateral ureteral obstruction. Nephrology (Carlton). 2007;12(6):565-571.

11. Pang M, Ma L, Gong R, et al. A novel STAT3 inhibitor, S3I-201, attenuates renal interstitial fibroblast activation and interstitial fibrosis in obstructive nephropathy. Kidney Int. 2010;78(3):257-268.

12. Walker SR, Chaudhury M, Nelson EA, Frank DA. Microtubule-targeted chemotherapeutic agents inhibit signal transducer and activator of transcription 3 (STAT3) signaling. Mol Pharmacol. 2010;78(5): 903-908.

13. Zhang D, Li Y, Liu Y, et al. Paclitaxel ameliorates lipopolysaccharideinduced kidney injury by binding myeloid differentiation protein-2 to block toll-like receptor 4-mediated nuclear factor- $\kappa \mathrm{B}$ activation and cytokine production. J Pharmacol Exp Ther. 2013;345(1):69-75.

14. Zhang D, Liu Y, Wei Q, et al. Tubular p53 regulates multiple genes to mediate AKI. J Am Soc Nephrol. 2014;25(10):2278-2289.

15. Grande MT, Lopez-Novoa JM. Fibroblast activation and myofibroblast generation in obstructive nephropathy. Nat Rev Nephrol. 2009;5(6): 319-328.

16. Siddiquee K, Zhang S, Guida WC, et al. Selective chemical probe inhibitor of Stat3, identified through structure-based virtual screening, induces antitumor activity. Proc Natl Acad Sci U S A. 2007;104(18): 7391-7396.

17. Yamamoto T, Matsuda T, Muraguchi A, et al. Cross-talk between IL-6 and TGF-beta signaling in hepatoma cells. FEBS Lett. 2001;492(3): 247-253.

18. Vij N, Sharma A, Thakkar M, et al. PDGF-driven proliferation, migration, and IL8 chemokine secretion in human corneal fibroblasts involve JAK2 STAT3 signaling pathway. Mol Vis. 2008;14(23):1020-1027.

19. Kretzschmar AK, Dinger MC, Henze C, et al. Analysis of Stat3 (signal transducer and activator of transcription 3) dimerization by fluorescence resonance energy transfer in living cells. Biochem J. 2004; $377: 289-297$.
20. Lim CP, Phan TT, Lim IJ, et al. Cytokine profiling and Stat3 phosphorylation in epithelial-mesenchymal interactions between keloid keratinocytes and fibroblasts. J Invest Dermatol. 2009;129(4):851-861.

21. Lane A, Johnson DW, Pat B, et al. Interacting roles of myofibroblasts, apoptosis and fibrogenic growth factors in the pathogenesis of renal tubulointerstitial fibrosis. Growth Factors. 2002;20 (3):109-119.

22. Vesey DA, Cheung CW, Cuttle L, et al. Interleukin-1beta induces human proximal tubule cell injury, alpha-smooth muscle actin expression and fibronectin production. Kidney Int. 2002;62(1):31-40.

23. Arakawa T, Masaki T, Hirai T, et al. Activation of signal transducer and activator of transcription 3 correlates with cell proliferation and renal injury in human glomerulonephritis. Nephrol Dial Transplant. 2008;23(11):3418-3426.

24. Berthier CC, Zhang H, Schin M, et al. Enhanced expression of Janus kinase-signal transducer and activator of transcription pathway members in human diabetic nephropathy. Diabetes. 2009;58(2):469-477.

25. Herrmann JE, Imura T, Song B, et al. STAT3 is a critical regulator of astrogliosis and scar formation after spinal cord injury. J Neurosci. 2008; 28(28):7231-7243.

26. Milanović D, Braun F, Weber W, et al. The influence of the combined treatment with Vadimezan (ASA404) and taxol on the growth of U251 glioblastoma xenografts. BMC Cancer. 2012;12:242.

27. Abubaker K, Luwor RB, Escalona R, et al. Targeted disruption of the JAK2/STAT3 pathway in combination with systemic administration of paclitaxel inhibits the priming of ovarian cancer stem cells leading to a reduced tumor burden. Front Oncol. 2014;4:75.

28. Stenvinkel P, Heimbürger O, Paultre F, et al. Strong association between malnutrition, inflammation, and atherosclerosis in chronic renal failure. Kidney Int. 1999;55(5):1899-1911.

29. Vidt DG. Inflammation in renal disease. Am J Cardiol. 2006;97(2A): 20A-27A.

30. Zhang $\mathrm{C}$, Li Y, Wu Y, et al. Interleukin-6/signal transducer and activator of transcription 3 (STAT3) pathway is essential for macrophage infiltration and myoblast proliferation during muscle regeneration. J Biol Chem. 2013;288(7):1489-1499.

31. Zeisberg M, Maeshima Y, Mosterman B, et al. Renal fibrosis. Extracellular matrix microenvironment regulates migratory behavior of activated tubular epithelial cells. Am J Pathol. 2002;160(6):2001-2008.

32. Lan HY. Tubular epithelial-myofibroblast transdifferentiation mechanisms in proximal tubule cells. Curr Opin Nephrol Hypertens. 2003; 12(1):25-29.
Drug Design, Development and Therapy

\section{Publish your work in this journal}

Drug Design, Development and Therapy is an international, peerreviewed open-access journal that spans the spectrum of drug design and development through to clinical applications. Clinical outcomes, patient safety, and programs for the development and effective, safe, and sustained use of medicines are a feature of the journal, which

\section{Dovepress}

has also been accepted for indexing on PubMed Central. The manuscript management system is completely online and includes a very quick and fair peer-review system, which is all easy to use. Visit $\mathrm{http}: / /$ www.dovepress.com/testimonials.php to read real quotes from published authors. 